\title{
Multispectral imaging system based on light-emitting diodes for the detection of melanomas and basal cell carcinomas: a pilot study (erratum)
}

\author{
Xana Delpueyo \\ Meritxell Vilaseca \\ Santiago Royo \\ Miguel Ares \\ Laura Rey-Barroso \\ Ferran Sanabria \\ Susana Puig \\ Josep Malvehy \\ Giovanni Pellacani \\ Fernando Noguero \\ Giuseppe Solomita \\ Thierry Bosch
}




\title{
Multispectral imaging system based on light-emitting diodes for the detection of melanomas and basal cell carcinomas: a pilot study (erratum)
}

\author{
Xana Delpueyo, ${ }^{a}$ Meritxell Vilaseca, ${ }^{a}$ Santiago Royo, ${ }^{a}$ Miguel Ares, ${ }^{a}$ Laura Rey-Barroso, ${ }^{\text {a }}$ Ferran Sanabria, ${ }^{a}$ \\ Susana Puig, ${ }^{b}$ Josep Malvehy,, Giovanni Pellacani, ${ }^{\mathrm{b}}$ Fernando Noguero, ${ }^{\mathrm{d}}$ Giuseppe Solomita, ${ }^{\mathrm{e}}$ and \\ Thierry Bosch ${ }^{f}$ \\ ${ }^{a}$ Technical University of Catalonia, Centre for Sensors, Instruments and Systems Development (CD6), Terrassa, Spain

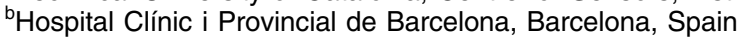 \\ 'Università di Modena e Reggio Emilia, Modena, Italy \\ ${ }^{\mathrm{d} C a r r i l}$ Instruments S.L., Barcelona, Spain \\ eMavig GmbH, Munich, Germany \\ Institut National Polytechnique de Tolouse, Toulouse, France
}

[DOI: $10.1117 / 1 . J B O \cdot 22.7 .079801]$

This article [J. Biomed. Opt. 22(6), 065006 (2017)] was originally published online on 29 June 2017. An author was accidentally omitted from the author list. Josep Malvehy contributed to the concept and design, data collection, analysis and interpretation, and obtained funding. He has been added to the author list as shown above.

This article was corrected online on 20 July 2017. 